\title{
Nanoscale investigation of Si nanoribbon growth on $\operatorname{Ag}(110)$
}

\author{
Michel Daher Mansour, Romain Parret and Laurence Masson ${ }^{\text {a) }}$ \\ Aix Marseille Univ, CNRS, CINaM, Campus de Luminy, Case 913, 13288 Marseille Cedex 9, France \\ a)Electronic mail: masson@cinam.univ-mrs.fr
}

We present a nanoscale investigation by means of scanning tunneling microscopy of Si nanostructure growth on the anisotropic silver (110) surface, in the submonolayer range. Four types of $\mathrm{Si}$ nanostructures are studied statistically as a function of the substrate temperature in the range $300 \mathrm{~K}$ to $500 \mathrm{~K}$ : isolated single- and double nanoribbons, which differ only by a factor of two in their width, and their respective bi-dimensional counterparts in the self-assembly regime. Our observations highlight different growth regimes controlled by kinetics. Below $320 \mathrm{~K}$, the $\mathrm{Si}$ adatoms diffuse along the easy [1 $\overline{1} 0$ ] direction, forming essentially isolated single nanoribbons randomly distributed on the silver terraces. At higher temperatures, transverse diffusion along the [001] direction is activated and a competition between the growth of self-assembled single nanoribbons and isolated double nanoribbons is observed. Above $440 \mathrm{~K}$, a transition from one- to two-dimensional double nanoribbon growth is evidenced. At $490 \mathrm{~K}$, the $\mathrm{Si}$ deposition results in the formation of massively self-assembled double nanoribbons. Based on Arrhenius analyses, activation barriers are found to be $(125 \pm$ $15) \mathrm{meV}$ and $(210 \pm 20) \mathrm{meV}$ for the formation of isolated Si nanoribbons and self-assembled Si double nanoribbons, respectively. Our results allow for a better understanding of the kinetic limiting processes which determine the submonolayer morphology and illustrate the role played by the missing row reconstruction of the $\mathrm{Ag}(110)$ surface in the formation of extended Si nanoribbon arrays. 


\section{INTRODUCTION}

Nanostructuring of surfaces using their intrinsic structural properties has received great interest over the last past two decades, with the aim at creating atomically controlled large scale templates. This step of nanopatterning is a promising route to produce by templatedirected growth high densities of true atomic dimension nanostructures with controlled geometries, regular sizes and spacings and specific physical properties related to their structural properties. The method applies for inorganic nanostructures and more recently for molecular arrangements. ${ }^{1-8}$ Moreover, the properties of interest of these highly dense and uniform surface-supported nanostructures such as electronic, optical or magnetic properties can be investigated using either local probes (scanning tunneling spectroscopy and spinpolarized scanning tunneling microscopy) or integrating techniques (angle-resolved photoelectron spectroscopy, surface differential reflectance spectroscopy and x-ray magnetic circular dichroism).

Growth proceeds in many cases away from thermodynamic equilibrium. The degree to which the growth occurs far from equilibrium conditions determines if the morphology of the grown structures is rather determined by thermodynamic quantities or by the growth kinetics. The kinetically controlled growth has proven to be, in the last twenty years, an alternative strategy to produce a wide variety of new zero-dimensional (0D) $)^{1,4}$, one-dimensional (1D) ${ }^{2}$ and two-dimensional (2D) ${ }^{9}$ nanostructures on substrates by playing with the growth conditions and the interplay between the adsorbate and the substrate. The morphology of these metastable nanostructures is the result of the competition between different thermally activated atomic scale processes characterized by their activation energy. Scanning tunneling microscopy (STM) has proven to be a technique of choice to obtain valuable information on the atomic structure of these nanostructures and the activated atomic scale processes taking place on surfaces. ${ }^{10,11}$ 
Among these nanostructures, 2D Si layers grown on silver substrates have recently attracted considerable interest for both their fundamental interest and expected promising properties analog to the electronic properties of graphene. ${ }^{12,13}$ It has been mainly reported since 2005 that submonolayer Si deposition on the anisotropic $\operatorname{Ag}(110)$ surface leads to the spontaneous formation of flat lying Si nanoribbons (NRs), perfectly aligned with the troughs of the bare (110) surface (see ${ }^{14-16}$ for the earliest articles). Si NRs with a width of $0.8 \mathrm{~nm}$ (denoted hereafter as single nanoribbons (SNRs)) are randomly distributed on silver terraces upon Si deposition at room temperature (RT) while larger Si NRs, $1.6 \mathrm{~nm}$ in width (denoted hereafter as double nanoribbons (DNRs)), self-assemble laterally to form regular DNR arrays with a pitch of $2 \mathrm{~nm}$ when the sample is heated at $\sim 470 \mathrm{~K}$. Surface diffraction experiments have shown that the SNRs are locally organized in a $3 \times 2$ superstructure when grown at $\sim 430$ K while a highly-ordered $5 \times 2$ superstructure composed of DNRs is formed upon Si deposition at higher temperature $(\sim 470 \mathrm{~K}) \cdot{ }^{17,18}$ Despite the expected simplicity of the Si/Ag interface, regarding the negligible miscibility of $\mathrm{Si}$ and $\mathrm{Ag}$ below $\sim 1070 \mathrm{~K}$, it took about 10 years to solve the atomic structure of these Si NRs. The first step was to evidence using STM and grazing incidence $x$-ray diffraction (GIXD) that the $\operatorname{Ag}(110)$ surface surprisingly undergoes a missing row reconstruction underneath the Si NRs during the growth of both SNRs and DNRs. ${ }^{18}$ An original surface-supported Si arrangement was later proposed by Cerdá et al. ${ }^{19}$, based on density functional theory (DFT), rapidly confirmed by Prévot et al. ${ }^{20}$ using a combined GIXD-STM-DFT study: the Si NRs correspond to pentamer chains lying in the missing row troughs of the reconstructed $\mathrm{Ag}(110)$ surface. Apart from these studies, the Si DNR array, covering uniformly the whole $\operatorname{Ag}(110)$ surface, has been successfully used as a template for the growth of identical 1D nanostructures made of $\mathrm{Co}^{21}, \mathrm{Mn}^{22}, 9,10$ phenanthrenequinone ${ }^{23}$ or $\mathrm{C}_{60}{ }^{24}$ and the study of their magnetic properties in the case of $\mathrm{Co}^{25}$. The use of this Si array as a template takes advantage of its high structural quality (low defect 
density at the atomic scale and single domain orientation of the Si grating) and the electronic decoupling of adsorbates from the conductive substrate, which is crucial to study the magnetic properties of supported nanostructures.

While the atomic structure of the Si NRs has been solved, the growth mechanisms of their arrangement in $3 \times 2$ and $5 \times 2$ superstructures have received little attention. However, a detailed knowledge of the atomic scale processes involved is required for understanding and controlling growth morphologies in the kinetic growth regime. The control of the formation of the Si superstructures would allow for the nanofabrication of highly-ordered templates with different geometries by varying the width of the $\mathrm{Si}$ NRs and the pitch of the grating. Moreover, questions remain regarding the early stages of growth, especially concerning the role and the atomic structure of the Si nanodots observed by STM, considered as the precursor for the formation of SNRs. ${ }^{19,26}$

Here, we present a nanoscale investigation of the $\mathrm{Si}$ NR growth on $\operatorname{Ag}(110)$, for submonolayer Si coverage and substrate temperature ranging from $300 \mathrm{~K}$ to $500 \mathrm{~K}$. The behavior of four types of Si nanostructures have been studied: isolated SNRs and isolated DNRs (denoted hereafter as 1D-SNRs and 1D-DNRs, respectively) and their respective 2D counterparts in the self-assembly regime (denoted hereafter as 2D-SNRs associated with the $3 \times 2$ superstructure and $2 \mathrm{D}$-DNRs associated with the $5 \times 2$ superstructure, respectively). We focus our study on the statistical analysis of the Si NR density measured in STM images to gain a better understanding of the kinetic limiting processes in the different growth modes observed. Our results strongly suggest that the transition from $\mathrm{Si}$ nano-dots to the NR structure is the limiting process for the growth of both 1D-SNRs and 1D-DNRs while the growth of 2D-SNRs and 2D-DNRs is driven by the formation of close-packed silver missing rows. 


\section{EXPERIMENT}

All the experiments were carried out in an ultrahigh vacuum (UHV) set-up. The (110) surface of a silver single-crystal was prepared by repeated cycles of $\mathrm{Ar}^{+}$sputtering and annealing at $770 \mathrm{~K}$. The cleanness of the $\mathrm{Ag}(110)$ surface was systematically checked by STM before Si deposition. Si was evaporated from a direct current heated piece of silicon wafer kept at $1520 \mathrm{~K}$ onto the clean $\mathrm{Ag}(110)$ surface, after preoutgassing at $1070 \mathrm{~K}$ for several hours. The clean $\operatorname{Ag}(110)$ sample was exposed to a Si flux at a constant deposition rate of $5 \times 10^{-4}$ monolayer (ML) per second. $1 \mathrm{ML}$ corresponds to the $\mathrm{Ag}(110)$ surface atom density, which also equals the density of the perfect $3 \times 2$ superstructure completely covering the $\operatorname{Ag}(110)$ surface. We focused our experiments on two low Si coverages $\left(\theta_{\mathrm{Si}} \sim 0.22\right.$ and $0.54 \mathrm{ML}$ ) to avoid coalescence of Si NR islands. The reproducibility of the deposition of this home-made $\mathrm{Si}$ evaporator is $\sim \pm 10 \%$. The substrate was heated at the rear side by radiation emitted by a commercial Scienta Omicron heater made of a $\mathrm{W}$ filament. The substrate temperature (T) during Si deposition was measured using a chromel-alumel thermocouple, spot welded very close to the Ag sample and calibrated prior to the set of experiments using a heating power-substrate temperature relationship established with the thermocouple spot welded instead of the Ag sample. The accuracy of the temperature measurement is $\pm 10 \mathrm{~K}$.

STM measurements were carried out at the CINaM in Marseille, using Scienta Omicron apparatus. All STM images were obtained at RT in the constant current mode with sample voltages from $-1.2 \mathrm{~V}$ to $-0.7 \mathrm{~V}$ and tunneling current of $\sim 300 \mathrm{pA}$. The $\mathrm{X}-\mathrm{Y}$ piezoelectric scanner of the STM was carefully calibrated using the extended $5 \times 2$ Si DNR superstructure. The STM data have been processed using the Gwyddion software. ${ }^{27}$ After Si deposition at substrate temperatures ranging from $300 \mathrm{~K}$ to $500 \mathrm{~K}$, the surface was frozen down to RT, where no motion or modification of the Si NRs is observed. The Si NR counting was performed from STM images with typical size $(300 \times 300) \mathrm{nm}^{2}$, insuring that SNRs and 
DNRs can be distinguished and the spacing between Si NRs can be measured. For each temperature and each Si coverage, at least 2 STM images have been used to perform a statistical analysis of the Si NRs. The typical number of Si NRs in one STM image, counted along the direction perpendicular to the Si NRs, is $\sim 70$ SNRs at low temperature and $\sim 35$ DNRs at high temperature, for $\theta_{\mathrm{Si}} \sim 0.22 \mathrm{ML}$. For each STM image, several measurements of the percentage of the different kinds of Si NRs formed and of the transverse linear density of Si NRs along the direction perpendicular to the NRs, have been performed using line profiles, except for the higher growth temperatures $(\mathrm{T}>420 \mathrm{~K}$ ), where the length of the Si NRs was longer than the size of STM images $(300 \mathrm{~nm}$ ) and thus only one measurement was possible. The error bar of the Si NR density is $\pm 10 \%$, corresponding to the mean statistical variation observed, for a given temperature, between the different measurements made. The lattice parameters of $\operatorname{Ag}(110)$ is $0.409 \mathrm{~nm}$ in the [001] direction, perpendicular to the Si NR axis and $0.289 \mathrm{~nm}$ in the [1ํㅣㅇㅣ direction, parallel to the Si NR axis.

GIXD experiments were carried out on the ID3 beamline of the European Synchrotron Radiation Facility (ESRF) storage ring with $17 \mathrm{keV}$ x-rays and the incidence angle kept fixed at $0.22^{\circ}$. The basis is expressed in the cubic basis of Ag, $\vec{a}_{x}=a(0,0,1), \vec{a}_{y}=a(1 / 2,-1 /$ $2,0)$ and $\vec{a}_{z}=a(1 / 2,1 / 2,0)$, where $a=0.409 \mathrm{~nm}$. The corresponding $(h, k, l)$ indices are used for indexing a reflection in reciprocal space.

\section{Results}

We have investigated the $\mathrm{Si}$ growth on $\mathrm{Ag}(110)$ for substrate temperatures ranging from $300 \mathrm{~K}$ to $500 \mathrm{~K}$ and in the submonolayer regime. For these growth conditions, the deposition of Si on $\mathrm{Ag}(110)$ leads to the formation of a Si ultrathin layer composed of Si NRs. Different arrangements of the Si NRs, depending on the growth temperature and for the lower coverage studied, can be viewed in the STM images of Fig. 1. At RT, 1D-SNRs are 
essentially formed and are randomly distributed on the silver terraces [Fig. 1(a)], as already reported. ${ }^{14,18} \mathrm{Si}$ nano-dots, which are considered to be the precursor for the formation of SNRs, are also visible. ${ }^{15,19,26}$ At $350 \mathrm{~K}$, as illustrated in Fig. 1(b), we observe that the SNRs markedly elongate - they can reach lengths up to $300 \mathrm{~nm}$ along the longitudinal [1 10$]$ direction - and that they start to agglomerate in the [001] direction. At $390 \mathrm{~K}$, as shown in Fig. 1(c), 2D-SNRs are locally organized in a $3 \times 2$ superstructure while 1 D-DNRs start to appear. Finally, at $480 \mathrm{~K}$, we observe the formation of the highly-ordered $5 \times 2$ superstructure [Fig. 1(d)], composed of 2D-DNRs, widely described in previous publications. ${ }^{16-18}$ Since the different geometries of the Si deposit observed by STM in our experiments differ essentially by the lateral organization of the Si NRs in the [001] direction, we have focused our statistical analysis on the linear density of Si NRs in the [001] direction (denoted hereafter as transverse linear density TLD). In Fig. 2(a), which shows the TLD of SNRs and DNRs for $\theta_{\mathrm{Si}} \sim 0.22$ ML, we clearly see the transition between the formation of SNRs and DNRs, occurring in the [370 K - $440 \mathrm{~K}]$ temperature range. Similar graph, not shown here, was obtained for $\theta_{\mathrm{Si}} \sim$ 0.54 ML. The ratio between the two plateaus is $\sim 2$, within the error bars, in agreement with recent theoretical structural models showing that DNRs correspond to twin Si pentamer chains, by a lateral repetition of the motif of SNRs. ${ }^{19}$ We can conclude that no other Si nanostructures are formed in the temperature range of interest. 

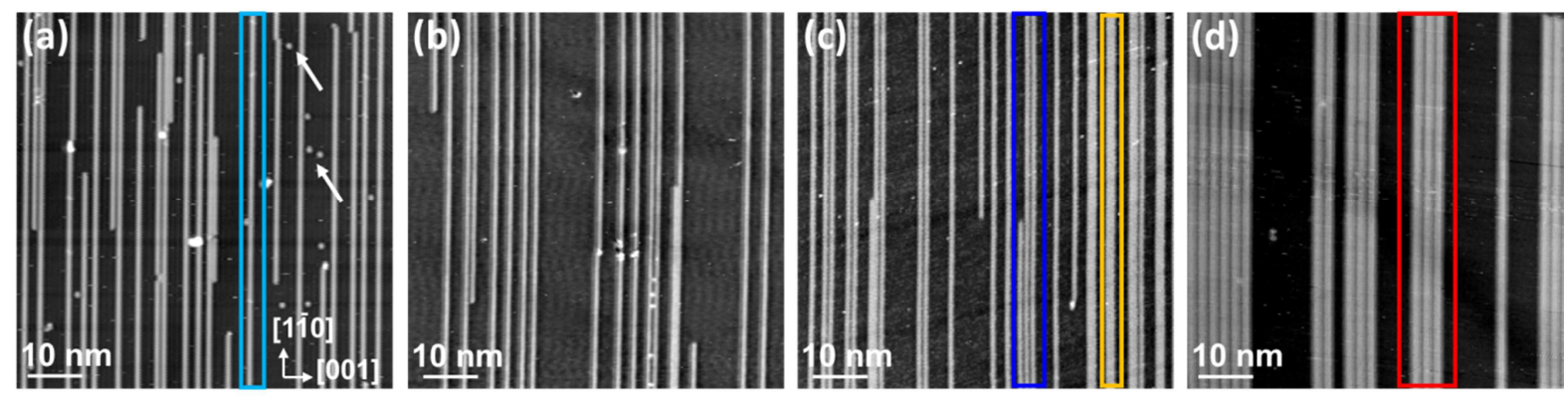

FIG. 1. (Color Online) STM images $\left((70 \times 70) \mathrm{nm}^{2}\right)$ of $\mathrm{Si}$ deposited on $\operatorname{Ag}(110)$ at four different substrate temperatures and nearly the same Si coverage $\theta_{\mathrm{Si}} \sim 0.22 \mathrm{ML}$, showing the formation of SNRs and DNRs : (a) RT, (b) $350 \mathrm{~K}$, (c) $390 \mathrm{~K}$ and (d) $480 \mathrm{~K}$. The four kinds of Si NRs are indicated by colored rectangles: 1D-SNRs (light blue in (a)) and 1D-DNRs (orange in (c), right side), 2D-SNRs (blue in (c), right middle part) and 2D-DNRs (red in (d)). In (a), the arrows indicate the formation of small Si nano-dots.

To get a deeper insight in the growth mechanisms of the Si NRs, we have plotted in Fig. 2(b), as a function of the substrate temperature during Si deposition, the percentage of the four Si species formed: 1D-SNRs, 1D-DNRs, 2D-SNRs and 2D-DNRs assembled in $3 \times 2$ and $5 \times 2$ arrangements, respectively, even locally (when only two SNRs or DNRs are assembled). Below $390 \mathrm{~K}$, mostly 1D-SNRs are formed with a slow increase of the percentage of 2DSNRs with the substrate temperature. At $390 \mathrm{~K}, 2 \mathrm{D}-\mathrm{SNRs}$ are the predominant species with an abrupt increase of the corresponding percentage while 1D-DNRs start to form. The formation of the $3 \times 2$ superstructure above $390 \mathrm{~K}$ is in agreement with the LEED observation reported in ${ }^{17}$ for silicon deposition at $\sim 430 \mathrm{~K}$. In this latter article, the broad spots associated with the $\times 3$ periodicity in the [001] direction indicate a short range formation of the $3 \times 2$ superstructure, similarly to our observations. Above $440 \mathrm{~K}, 2 \mathrm{D}-\mathrm{SNRs}$ are no longer formed and 1D-DNRs are replaced by 2D-DNRs. Finally, at $490 \mathrm{~K}$, DNRs are massively assembled to form 2D-DNR domains. 


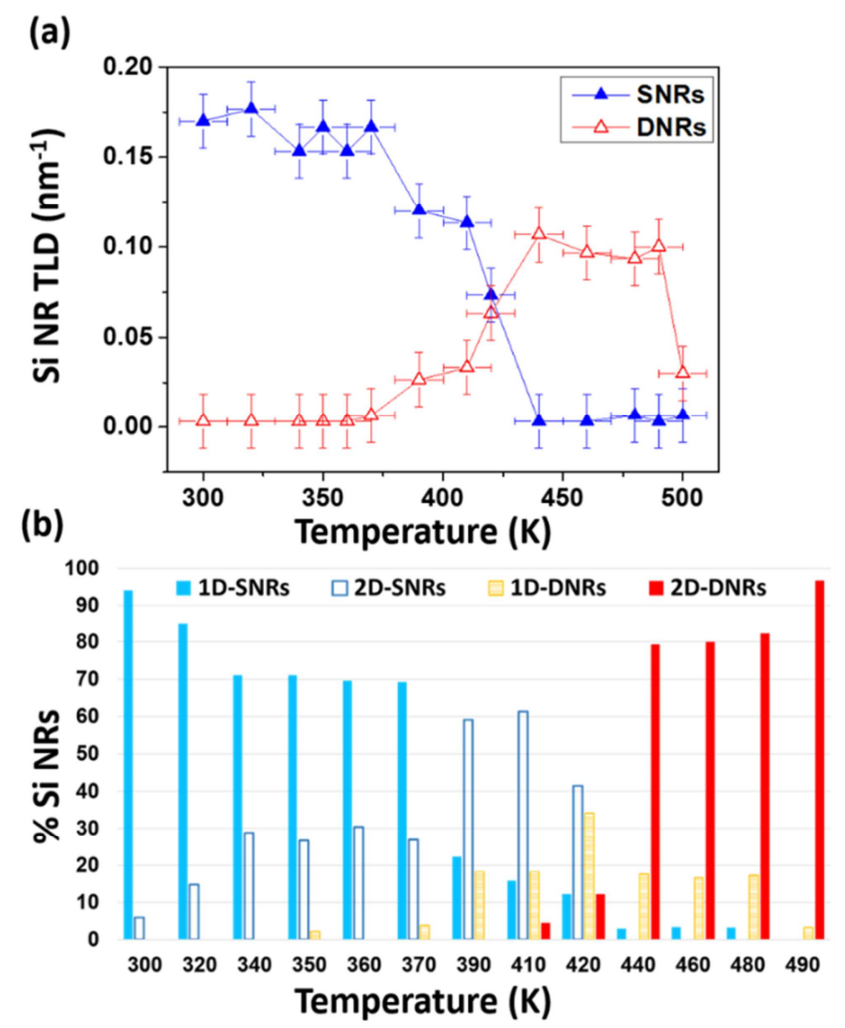

FIG. 2. (Color Online) (a) Transverse linear density (TLD) of Si NRs, perpendicular to the NRs (blue filled triangles and red open triangles) as a function of the substrate temperature for $\theta_{\mathrm{Si}} \sim 0.22 \mathrm{ML}$. (b) Corresponding percentages of 1D-NRs, 1D-DNRs, 2D-NRs and 2D-DNRs for each substrate temperature.

Considering that the formation of the four Si species obeys to Arrhenius laws, we have interpreted our STM images by analyzing the density of anisotropic Si islands as a function of the substrate temperature, as frequently reported in the literature for both isotropic 2D islands and anisotropic 1D-2D islands. ${ }^{10,28}$ In Figs. 3(a) and 3(b), we have plotted the TLD of SNR islands and DNR islands as a function of $1 / \mathrm{T}$ for $0.22 \mathrm{ML}$ and $0.54 \mathrm{ML}$. SNR islands correspond to either 1D-SNRs or 2D-SNRs and DNR islands to either 1D-DNRs or 2DDNRs. In Fig. 3(a), the surface density of NRs is also plotted in the lower temperature range [300 K - $340 \mathrm{~K}$ ], for which the 2D counting of Si NRs is possible using STM images $\left(300 \times 300 \mathrm{~nm}^{2}\right)$. For $\mathrm{T} \leq 340 \mathrm{~K}$, this overall density shows Arrhenius behavior with an 
activation energy of $(125 \pm 15) \mathrm{meV}$, showing that the longitudinal Si adatom diffusion, along the easy [1 $1 \overline{1} 0]$ direction, is already active for the lowest temperatures of our experiments. The plateau observed above $340 \mathrm{~K}$ illustrates that most of the Si NRs have reached more than 300 nm in length. In Figs. 3(a) and 3(b), the TLD of SNR islands remains almost constant below $370 \mathrm{~K}$. The break in the TLD, for both coverages, between $320 \mathrm{~K}$ and $340 \mathrm{~K}$, can be attributed to Si adatom transverse migration, along the [001] direction, leading to a local packing of the 1D-SNRs. Above $370 \mathrm{~K}$, we observe an abrupt decrease of the SNR island TLD due to either the formation of 2D-SNRs composed of $\sim 3$ SNRs or the formation of 1D-DNRs. Till $430 \mathrm{~K}$, there is a clear competition between the formation of both $\mathrm{Si}$ arrangements. At higher temperature (above $440 \mathrm{~K}$ ), the break of the DNR island TLD for both coverages clearly reflects the transition from 1D-DNR to 2D-DNR growth with an activation energy of $(210 \pm$ 20) $\mathrm{meV}$.

(a)

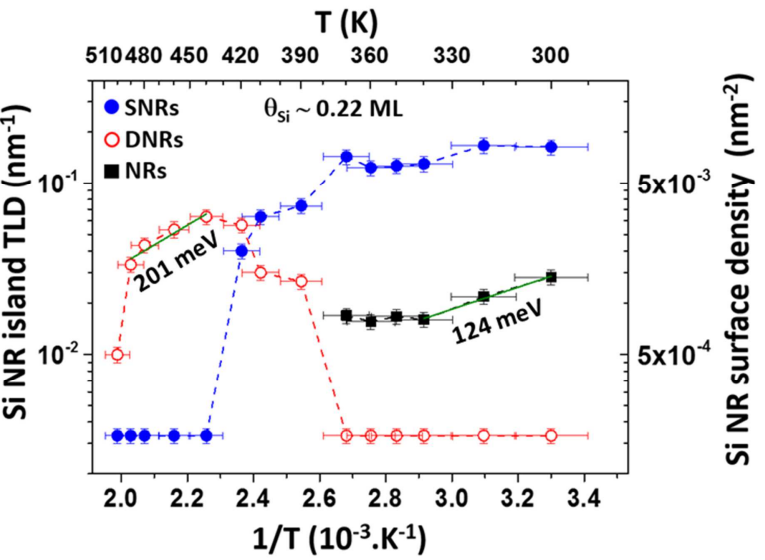

(b)

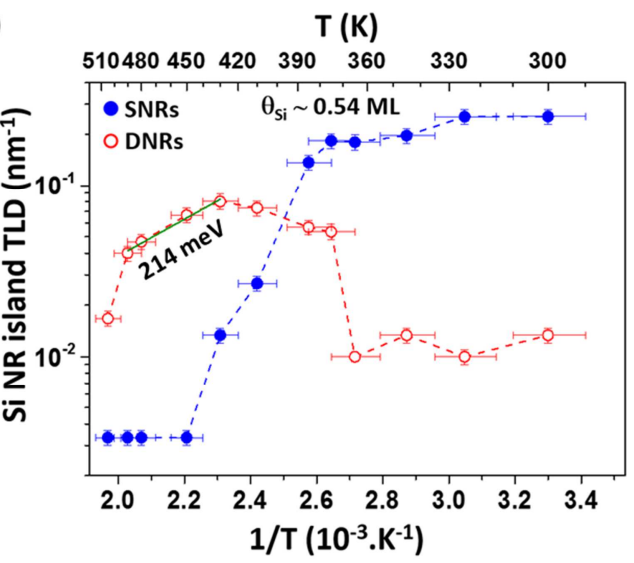


FIG. 3. (Color Online) (a) Arrhenius plot of the transverse linear density (TLD) of Si SNR islands (blue filled dots) and Si DNR islands (red open dots), in $\mathrm{nm}^{-1}$, and the surface density of Si NRs (black dots), in $\mathrm{nm}^{-2}$, for $\theta_{\mathrm{Si}} \sim 0.22 \mathrm{ML}$ as a function of $1 / \mathrm{T}$. (b) Arrhenius plot of the transverse linear density (TLD) of Si SNR islands (blue filled dots) and Si DNR islands (red open dots), in $\mathrm{nm}^{-1}$, for $\theta_{\mathrm{Si}} \sim 0.54 \mathrm{ML}$ as a function of $1 / \mathrm{T}$.

Above $490 \mathrm{~K}$, we observe a strong decrease of the Si NR TLD measured by STM. This behavior has also been observed by GIXD as illustrated in Fig. 4. In Fig. 4(a), the evolution of the Si NR TLD, expressed in SNRs/nm (i.e. each DNR is counted twice) is reported for $\theta_{\mathrm{Si}} \sim 0.22 \mathrm{ML}$ and $0.54 \mathrm{ML}$, as a function of the substrate temperature during $\mathrm{Si}$ deposition. Figure 4(b) displays the behavior, as a function of the annealing substrate temperature, of the intensity of three intense GIXD diffraction peaks associated with the $\times 5$ periodicity of the $5 \times 2$ superstructure at completion (see ${ }^{18}$ for more details concerning the GIXD experiments). This abrupt break at $490 \mathrm{~K}$ corresponds to a drastic change of the morphology of the $\mathrm{Si} / \mathrm{Ag}$ interface, described $\mathrm{in}^{29}$ as a silver surface faceting induced by $\mathrm{Si}$ adatoms.

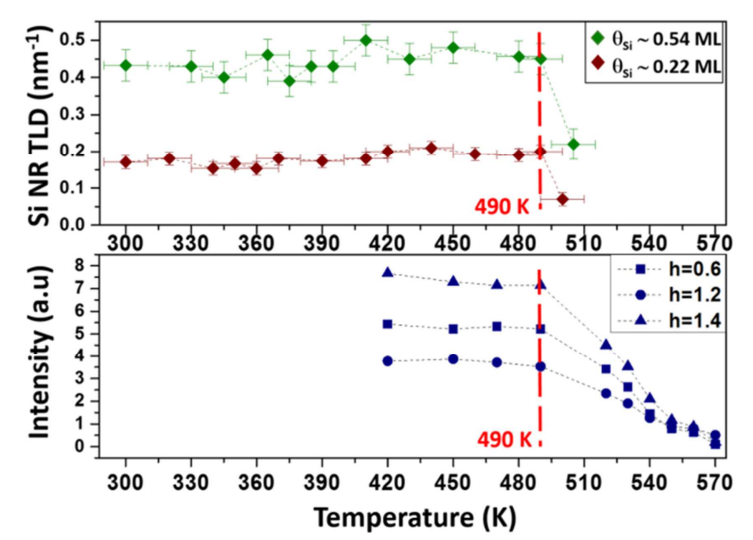

FIG. 4. (Color Online) (a) : transverse linear density (TLD) of Si NRs, expressed in SNRs/nm, as a function of the substrate temperature during $\mathrm{Si}$ deposition for $\theta_{\mathrm{Si}} \sim 0.22 \mathrm{ML}$ and 0.54 
ML. (b) : evolution, as a function of the annealing substrate temperature, of the GIXD diffracted intensities associated with the $5 \times 2$ DNR superstructure for $h=0.6$ (blue squares), $h=1.2$ (blue dots), $h=1.4$ (blue triangles), $k=0$ and $l=0.05$. The intensity of the $(1.4,0,0.05$ ) reflection is divided by a factor of 5 .

\section{IV.DISCUSSION}

In our experiments, we clearly observe two transitions from 1D to $2 \mathrm{D}$ growth of anisotropic islands: (i) in the middle temperature range, with the formation of 1D-SNRs and 2D-SNRs self-assembled in a $3 \times 2$ superstructure above $370 \mathrm{~K}$ and (ii) in the higher temperature range, with the formation of $1 \mathrm{D}-\mathrm{DNR}$ and $2 \mathrm{D}$-DNRs self-assembled in a $5 \times 2$ superstructure above $440 \mathrm{~K}$. Similar transition has been reported $\mathrm{in}^{28}$ in the growth of anisotropic $\mathrm{Cu}$ islands on $\mathrm{Pd}(110)$. This transition was attributed to the anisotropy of diffusion along and across the easy [1 $1 \overline{1} 0]$ direction. In our system, the transverse migration is activated above $320 \mathrm{~K}$ but it doesn't lead to the formation of $3 \times 2$ islands, which is observed above 370 K. The same behavior occurs at higher temperature with the formation of 1D-DNRs above $370 \mathrm{~K}$ and $5 \times 2$ islands above $440 \mathrm{~K}$. This clearly demonstrates that the formation of the $\mathrm{Si}$ species on $\operatorname{Ag}(110)$ is driven by the activation barriers for their formation rather than by the diffusion of Si adatoms. From our results, we can easily rank from the lowest to the highest the activation energy for the formation of 1D-SNRs, 2D-SNRs, 1D-DNRs and 2D-DNRs.

Two models have been proposed for the structure of the Si nano-dot considered as the precursor of Si SNRs: one has a quasi-hexagonal geometry and the other corresponds to two

Si pentagons sitting side by side, both structures are located in a Ag di-vacancy. ${ }^{19,26}$ In both articles, a transition from unstable cluster precursors to SNRs is mentioned, when more Ag atoms are removed to form a trough. Let us remind that a DNR corresponds to not-linked twin Si SNRs running along a silver missing row. STM observations, not shown here, performed 
after $\mathrm{Si}$ growth at $490 \mathrm{~K}$, when a highly perfect $5 \times 2$ superstructure is formed, reveal that when a DNR has nucleated, the twin Si SNRs grow simultaneously as the termination of one DNR corresponds to the terminations of both twin SNRs. This strongly suggests that double $\mathrm{Si}$ nano-dots can be considered as the precursor structure for the formation of DNRs. We can thus assess that the transition from Si nano-dot to NR structure is the limiting process for the growth of both 1D-SNRs and 1D-DNRs, with a higher activation barrier for the formation of double Si nano-dots. Single Si nano-dots, precursor structure of the Si SNRs, are frequently observed by STM, for instance after growth at temperatures slightly higher than RT. To our knowledge, observation of double Si nano-dots has never been reported. Their observation by STM is rather challenging as they statistically constitute very rare events for Si growth at low temperature and would require a fast cooling of the sample after growth at high temperature to quench any atomic scale processes occurring on the surface.

One can wonder why a transition from 1D to 2D NR growth doesn't occur once the transverse diffusion of $\mathrm{Si}$ adatoms is activated. One argument can be given if we consider the $3 \times 2$ and $5 \times 2$ superstructures, which have a silver missing row TLD of $1 / 3$ and $2 / 5$, respectively and remark that the denser $2 \times 2$ superstructure made of pentamer chains with a silver missing row TLD of $1 / 2$ is not formed. This indicates that the formation of closepacked silver missing rows is a limited process for the formation of self-assembled $\mathrm{Si}$ pentamer chains. Between $370 \mathrm{~K}$ and $440 \mathrm{~K}$, we observe a competition between the formation of local $3 \times 2$ arrangements composed of $\sim 3$ SNRs and 1D-DNRs. This occurs when the number of close-packed silver missing rows of the local $3 \times 2$ arrangement induces an activation barrier higher than the one for the formation of 1D-DNRs. We can note that this transition occurs when the number of Ag missing rows of the $3 \times 2$ structure is higher than the one of 1D-DNRs, with similar spacing. This could explain why no extended $3 \times 2$ superstructures can be formed on $\mathrm{Ag}(110)$. 
Finally, the presence in STM images of SNRs evolving in DNRs (or vice versa) during the growth of Si NRs, as observed in Fig. 1(a), 1(b) or 1(c), has to be discussed. These features could result from the coalescence of growing SNRs and DNRs along the [1 $\overline{1} 0]$ direction, parallel to the NRs. Another explanation could be that a DNR evolves in a SNR due to the activation barrier for the formation of 2 close-packed silver missing rows. Lastly, let us consider that a SNR could evolve in a DNR. One mechanism could be that a single Si nanodot nucleates in the close vicinity of a SNR and leads to the growth of a twin SNR, forming thus a DNR. This mechanism is not consistent with the formation of double Si nano-dots, precursor for the formation of DNRs. Our STM observations made after Si growth at $490 \mathrm{~K}$ related to the termination of DNRs and the formation of 2D-SNRs in a large temperature range are in favor of the formation of double Si nano-dots. Nevertheless, we cannot exclude the mechanism of nucleation of single Si nano-dots in the close vicinity of SNRs to explain the formation of DNRs.

\section{CONCLUSION}

We have reported a nanoscale study of the Si NR growth which allows for a better description and understanding of the different growth regimes in the submonolayer range. Our results underline the role played by the silver substrate in the growth mechanisms occurring upon $\mathrm{Si}$ deposition. This highlights the strong interaction between $\mathrm{Si}$ and $\mathrm{Ag}$ in the formation of the surface-supported Si NRs, already reported for their structural ${ }^{20,30}$, electronic $^{31}$ and optical properties $^{32,33}$. We show that, due to the competition between the growth of 2D-SNRs and 1D-DNRs, no extended self-assembled SNR array can be formed, in contrary to the case of self-assembled DNRs. The former array has thus a limited reliability for its use as a 1D template, in contrary to the latter one. In the temperature range where only one type of Si arrangement is observed, the activation barrier for the formation of 1D-SNRs and 2D-DNRs 
are extracted from the measurement of $\mathrm{Si} \mathrm{NR}$ densities as a function of the substrate temperature. A detailed analysis of these densities using mean-field nucleation theory and kinetic Monte Carlo simulations would provide a profound understanding of the atomic scale processes involved and information about the precursor structure for the formation of Si NRs, which is still in debate.

\section{ACKNOWLEDGMENTS}

The authors gratefully acknowledge Geoffroy Prévot and Romain Bernard from the INSP (Paris) for fruitful discussions and collaboration during the GIXD run performed on the ID3 beamline at ESRF (Grenoble). Support from the ID3 beamline staff is acknowledged.

1. H. Brune, M. Giovannini, K. Bromann and K. Kern, Nature 394, 451 (1998).

2. P. Gambardella, A. Dallmeyer, K. Maiti, M. C. Malagoli, W. Eberhardt, K. Kern and C. Carbone, Nature 416, 301 (2002).

3. H. Ellmer, V. Repain, M. Sotto and S. Rousset, Sur. Sci. 511, 183 (2002).

4. N. Weiss, T. Cren, M. Epple, S. Rusponi, G. Baudot, S. Rohart, A. Tejeda, V. Repain, S. Rousset, P. Ohresser, F. Scheurer, P. Bencok and H. Brune, Phys. Rev. Lett. 95, 157204 (2005).

5. E. Cañas-Ventura Marta, W. Xiao, D. Wasserfallen, K. Müllen, H. Brune, V. Barth Johannes and R. Fasel, Angew. Chem. 46, 1814 (2007).

6. S. Clair, S. Pons, H. Brune, K. Kern and V. Barth Johannes, Angew. Chem. 44, 7294 (2005).

7. Q. Fan, J. Dai, T. Wang, J. Kuttner, G. Hilt, J. M. Gottfried and J. Zhu, ACS Nano 10, 3747 (2016). 8. P. Ruffieux, K. Palotás, O. Gröning, D. Wasserfallen, K. Müllen, W. A. Hofer, P. Gröning and R. Fasel, J. Am. Chem. Soc. 129, 5007 (2007).

9. R. Bernard, Y. Borensztein, H. Cruguel, M. Lazzeri and G. Prévot, Phys. Rev. B 92, 045415 (2015).

10.H. Brune, G. S. Bales, J. Jacobsen, C. Boragno and K. Kern, Phys. Rev. B 60, 5991 (1999).

11.D. Albertini, F. Thibaudau, L. Masson and F. Salvan, Surf. Sci. 400, 109 (1998).

12.P. De Padova, C. Quaresima, C. Ottaviani, P. M. Sheverdyaeva, P. Moras, C. Carbone, D. Topwal, B. Olivieri, A. Kara, H. Oughaddou, B. Aufray and G. Le Lay, Appl. Phys. Lett. 96, 261905 (2010). 13.P. Vogt, P. De Padova, C. Quaresima, J. Avila, E. Frantzeskakis, M. C. Asensio, A. Resta, B. Ealet and G. Le Lay, Phys. Rev. Lett. 108, 155501 (2012). 
14.C. Leandri, G. L. Lay, B. Aufray, C. Girardeaux, J. Avila, M. E. Dávila, M. C. Asensio, C. Ottaviani and A. Cricenti, Surf. Sci. 574, L9 (2005).

15.F. Ronci, S. Colonna, A. Cricenti, P. De Padova, C. Ottaviani, C. Quaresima, B. Aufray and G. Le Lay, Phys. Status Solidi C 7, 2716 (2010).

16.H. Sahaf, L. Masson, C. Léandri, B. Aufray, G. Le Lay and F. Ronci, Appl. Phys. Lett. 90, 263110 (2007).

17.S. Colonna, G. Serrano, P. Gori, A. Cricenti and F. Ronci, J. Phys.: Condens. Matter 25, 315301 (2013).

18.R. Bernard, T. Leoni, A. Wilson, T. Lelaidier, H. Sahaf, E. Moyen, L. Assaud, L. Santinacci, F. Leroy, F. Cheynis, A. Ranguis, H. Jamgotchian, C. Becker, Y. Borensztein, M. Hanbücken, G. Prévot and L. Masson, Phys. Rev. B 88, 121411(R) (2013).

19.J. I. Cerdá, J. Sławińska, G. Le Lay, A. C. Marele, J. M. Gómez-Rodríguez and M. E. Dávila, Nature Commun. 7, 13076 (2016).

20.G. Prévot, C. Hogan, T. Leoni, R. Bernard, E. Moyen and L. Masson, Phys. Rev. Lett. 117, 276102 (2016); G. Prévot, C. Hogan, T. Leoni, R. Bernard, E. Moyen and L. Masson, Phys. Rev. Lett. 118, 049901 (2017).

21.F. Dettoni, H. Sahaf, E. Moyen, L. Masson and M. Hanbücken, Europhys. Lett. 94, 28007 (2011).

22.P. De Padova, C. Ottaviani, F. Ronci, S. Colonna, B. Olivieri, C. Quaresima, A. Cricenti, M. E. Dávila, F. Hennies, A. Pietzsch, N. Shariati and G. Le Lay, J. Phys.: Condens. Matter 25, 014009 (2013).

23.E. Salomon and A. Kahn, Surf. Sci. 602, L79 (2008).

24.L. Masson, H. Sahaf, P. Amsalem, F. Dettoni, E. Moyen, N. Koch and M. Hanbücken, Appl. Surf. Sci. 267, 192 (2013).

25.L. Michez, K. Chen, F. Cheynis, F. Leroy, A. Ranguis, H. Jamgotchian, M. Hanbücken and L. Masson, Beilstein J. Nanotechnol. 6, 777 (2015).

26.S. Sheng, R. Ma, J.-b. Wu, W. Li, L. Kong, X. Cong, D. Cao, W. Hu, J. Gou, J.-W. Luo, P. Cheng, P.-H. Tan, Y. Jiang, L. Chen and K. Wu, Nano Lett. 18, 2937 (2018).

27.D. Nečas and P. Klapetek, Cent. Eur. J. Phys. 10, 181 (2012).

28.J. P. Bucher, E. Hahn, P. Fernandez, C. Massobrio and K. Kern, Europhys. Lett. 27, 473 (1994).

29.F. Ronci, G. Serrano, P. Gori, A. Cricenti and S. Colonna, Phys. Rev. B 89, 115437 (2014).

30.P. Lagarde, M. Chorro, D. Roy and N. Trcera, J. Phys.: Condens. Matter 28, 075002 (2016).

31.P. Gori, O. Pulci, F. Ronci, S. Colonna and F. Bechstedt, J. Appl. Phys. 114, 113710 (2013).

32.Y. Borensztein, G. Prévot and L. Masson, Phys. Rev. B 89, 245410 (2014); Y. Borensztein, G.

Prévot and L. Masson, Phys. Rev. B 96, 039904 (2017).

33.E. Speiser, B. Buick, N. Esser, W. Richter, S. Colonna, A. Cricenti and F. Ronci, Appl. Phys. Lett. 104, 161612 (2014). 